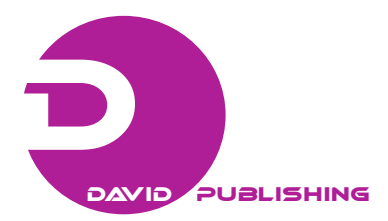

\title{
Simulation, Construction and Characterization of a Piezoelectric Transducer Using Rexolite as Acoustic Matching
}

\author{
Israel Sánchez Domínguez ${ }^{1,2}$, Pedro Acevedo Contla ${ }^{2}$, Manuel Recuero López ${ }^{1}$ and Demetrio Fabián García \\ Nocetti $^{2}$ \\ 1. Polytechnic University of Madrid, School of Industrial Engineering, Madrid, Spain \\ 2. National Autonomous University of Mexico, Institutito Research in Applied Mathematics and Systems, Mexico City, Mexico
}

Received: August 10, 2012 / Accepted: September 14, 2012 / Published: April 25, 2013.

\begin{abstract}
This article describes the simulation and characterization of an ultrasonic transducer using a new material called Rexolite to be used as a matching element. This transducer was simulated using a commercial piezoelectric ceramic PIC255 at $8 \mathrm{MHz}$. Rexolite, the new material, presents an excellent acoustic matching, specially in terms of the acoustic impedance of water. Finite elements simulations were used in this work. Rexolite was considered as a suitable material in the construction of the transducer due to its malleability and acoustic properties, to validate the simulations a prototype transducer was constructed. Experimental measurements were used to determine the resonance frequency of the prototype transducer. Simulated and experimental results were very similar showing that Rexolite may be an excellent matching, particularly for medical applications
\end{abstract}

Key words: Ultrasonic transducer, Rexolite, simulation, finite elements method.

\section{Introduction}

The good performance of a transducer is very important in all applications, in this case, the material that is used as matching plays a fundamental part, since if this material does not offer a good matching, the signal delivered by the transducer can be affected. The transmitted energy between the ultrasonic transducer and some means of transmission (e.g., blood in vessels or water) is maximized when the medium and the transducer have the same acoustic impedance.

Any impedance mismatch in the generation of ultrasound causes that the generated energy is reflected back to the transducer producing lost or leaks due to the absorption of the rear face of the transducer. A matching layer is a passive layer, which is added to the front of an ultrasonic transducer in order to improve the

Corresponding author: Israel Sánchez Domínguez, Ph.D. Student. E-mail: israel@uxdea4.iimas.unam.mx. matching of energy from and to the means of transmission.

In accordance with the conditions of a narrow band, the matching is maximized when the thickness of the layer is equal to a quarter of a wavelength (or a multiple of a quarter of a wavelength) of the energy that is transmitted (Fig. 1).

Research in the area of materials has progressed up to the stage of getting new acoustic materials with an acoustic impedance very similar to that of water, which helps perfectly in the transmission of energy between these two means.

The objective of this paper is to present a newmaterial, which has acoustic properties similar to those of water [1-3]. The finite element method was used in the simulation to determine the performance of the transducer constructed using Rexolite. Physical properties of Rexolite may be found in the following Refs. $[4,5]$. 


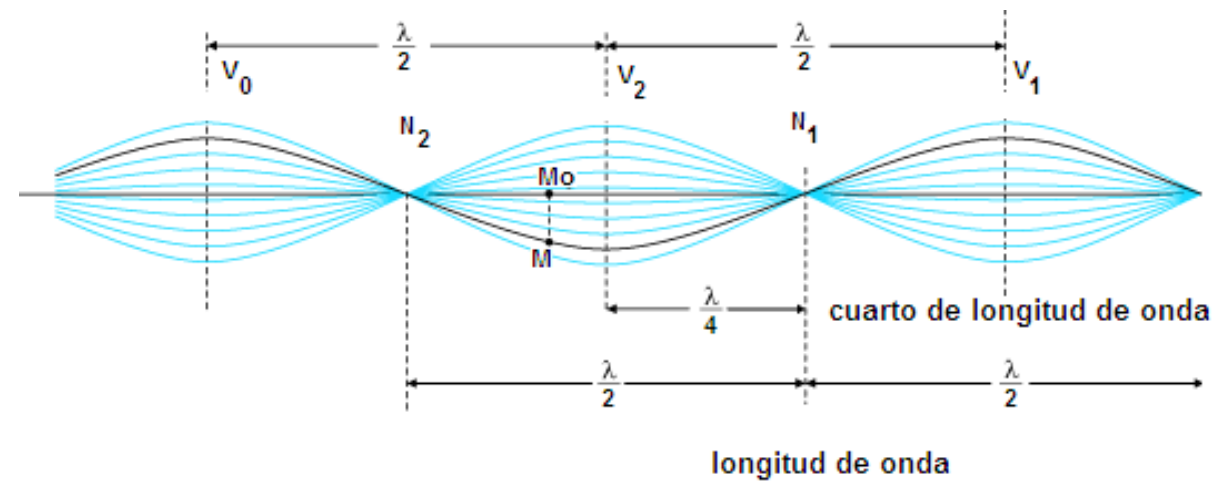

Fig. 1 Quarter wavelength concept.

\section{Materials and Methods}

Simulations were made considering a thin layer of the new material adhered to the top of the Piezoelectric ceramic, this was excited to observe the response of the ceramic, the Rexolite and both of them. All work was developed using the finite element method. Table No.1 shows the physical properties of the Piezoelectric ceramic PIC 255 at $8 \mathrm{MHz}$ [6]. Within the simulation work it is necessary to introduce to the program, matrices that allow a better modeling, such matrices are obtained from properties of the ceramics or even from materials used in the construction of these kind of arrays. Some of the parameters that are used to construct such arrays, for example, the Young's module, Poisson's coefficient, density, etc..

\subsection{Piezoelectric Ceramics}

According to previous work [1], the selected piezoelectric ceramic was the PIC255 at $8 \mathrm{MHz}$, which has square shape and the following dimensions $4.3 \mathrm{~mm}$ $\times 4.3 \mathrm{~mm} \times 0.3 \mathrm{~mm}$.

\subsection{Acoustic Coupling Material (Matching)}

As acoustic matching layer material, Rexolite 4422 was chosen, this material has an acoustic impedance similar to that of the medium of propagation [7-9], so there is a good transfer of energy between them.

Fig. 2 shows a photograph of the transducer with the current Rexolite layer. In this figure, the Rexolite layer used as an acoustic matching layer is shown, this material due to its acoustic properties is perfect to be used in ultrasonic transducers since we can ensure that there is maximum energy transfer through the Rexolite.

The way in which this layer was mounted it was placing a Rexolite cap on the transducer body and then stick the top part using Cyanoacrylate, this part has a notch that is attached to the soldering point allowing a homogeneous adherence which produces a sealed device with maximum energy transfer.

Table 1 Physical properties of the PIC255 piezoelectric ceramic.

\begin{tabular}{|c|c|c|c|c|}
\hline \multicolumn{5}{|c|}{ Material type PIC 255} \\
\hline \multicolumn{2}{|c|}{ Parameter } & & & \\
\hline \multicolumn{5}{|c|}{ Physical and dielectric properties } \\
\hline \multicolumn{2}{|c|}{ Density } & \multicolumn{2}{|l|}{$\rho\left(\mathrm{g} / \mathrm{cm}^{3}\right)$} & 7.80 \\
\hline \multicolumn{2}{|c|}{ Curie temperature } & \multicolumn{2}{|l|}{$\mathrm{T}_{\mathrm{c}}\left({ }^{\circ} \mathrm{C}\right)$} & 250 \\
\hline \multirow[b]{2}{*}{ Permittivity } & \multirow{2}{*}{$\begin{array}{l}\text { In the } \\
\text { polarization } \\
\text { direction } \\
\text { Perpendicular to } \\
\text { the polarity }\end{array}$} & \multicolumn{2}{|l|}{$\varepsilon_{33}{ }^{\mathrm{T}} / \varepsilon_{0}$} & 2,400 \\
\hline & & \multicolumn{2}{|l|}{$\varepsilon_{33}{ }^{\mathrm{T}} / \varepsilon_{0}$} & 1,980 \\
\hline \multicolumn{2}{|c|}{ Dielectric loss factor } & \multicolumn{2}{|c|}{$\tan \delta\left(10^{-3}\right)$} & 20 \\
\hline \multicolumn{5}{|c|}{ Electromechanical properties } \\
\hline \multirow{5}{*}{\multicolumn{2}{|c|}{ Coupling factors }} & $\mathrm{k}_{\mathrm{p}}$ & & 0.62 \\
\hline & & $\mathrm{k}_{\mathrm{t}}$ & & 0.53 \\
\hline & & $\mathrm{k}_{31}$ & & 0.38 \\
\hline & & $\mathrm{k}_{33}$ & & 0.69 \\
\hline & & $\mathrm{k}_{15}$ & & - \\
\hline \multirow{3}{*}{\multicolumn{2}{|c|}{ Piezoelectric charge constants }} & $d_{31}$ & \multirow{3}{*}{$\begin{array}{l}\left(10^{-12} \mathrm{C} /\right. \\
\mathrm{N})\end{array}$} & -210 \\
\hline & & $d_{33}$ & & 500 \\
\hline & & $\mathrm{d}_{15}$ & & - \\
\hline \multirow{2}{*}{\multicolumn{2}{|c|}{ Piezoelectric voltage constants }} & $\mathrm{g}_{31}$ & \multirow{2}{*}{$\begin{array}{l}\left(10^{-3}\right. \\
\left.\mathrm{V}_{\mathrm{m}} / \mathrm{N}\right)\end{array}$} & -11.5 \\
\hline & & $\mathrm{g}_{33}$ & & 22 \\
\hline
\end{tabular}




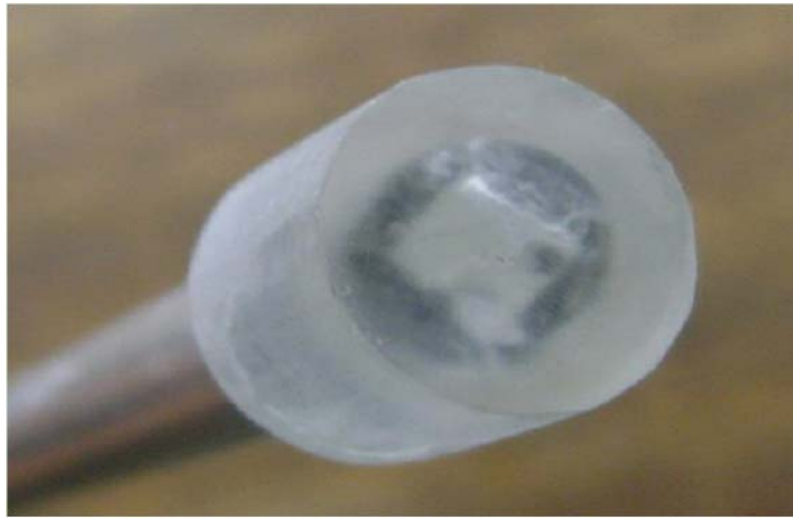

Fig. 2 Rexolite layer mounted on the transducer.

The characterization of the prototype transducer was using an Agilent 4294 Impedance Analyzer to determine the resonance frequency and to see the signal waveform an oscilloscope Tektronik TDS420 was used. The scan rate was from 4 to $10 \mathrm{MHz}$.

To obtain the value of the resonance frequency on an experimental basis, the equivalent circuit (shown in the Fig. 3) was replaced by the actual transducer, then a function generator was used, varying the frequency up to point of finding the minimum value of output voltage $(\mathrm{Vo})$ and hence, the lowest impedance of the transducer, which is the resonance frequency. This configuration is shown in Fig. 4.

\section{Results and Discussion}

Firstly, in Fig. 5 the results obtained in the simulation of the piezoelectric ceramics using COMSOL [10] are shown. Fig. 6 shows the impedance of the simulated piezoelectric ceramic.

The frequency obtained in the simulation corresponds to that of the ceramic (Fig. 6), comparing these two values we demonstrate that the simulation was correct. Now, continuing with the simulation, we add to the ceramic a thin film of Rexolite, allowing the understanding of the performance of the transducer once the piezoelectric ceramic and the Rexolite are bonded.

Fig. 7 shows the simulation of the piezoelectric ceramic and the Rexolite layer, only a piece of the total geometry is considered due to the fact that the simulation
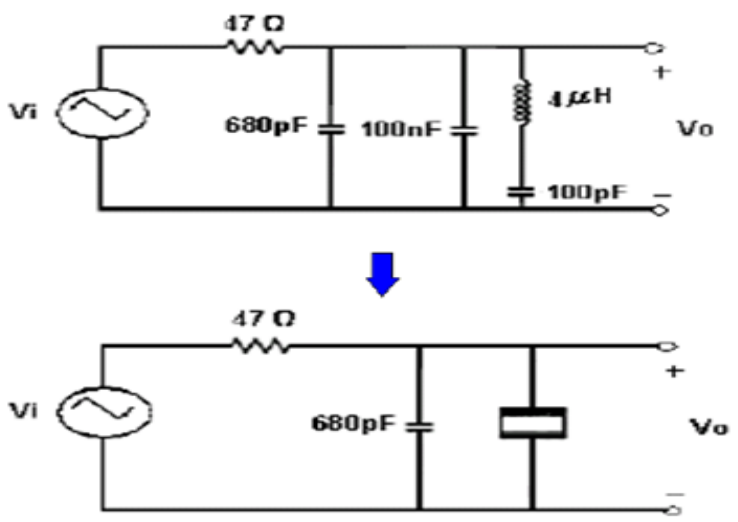

Fig. 3 Theoretical model and real transducer circuit.

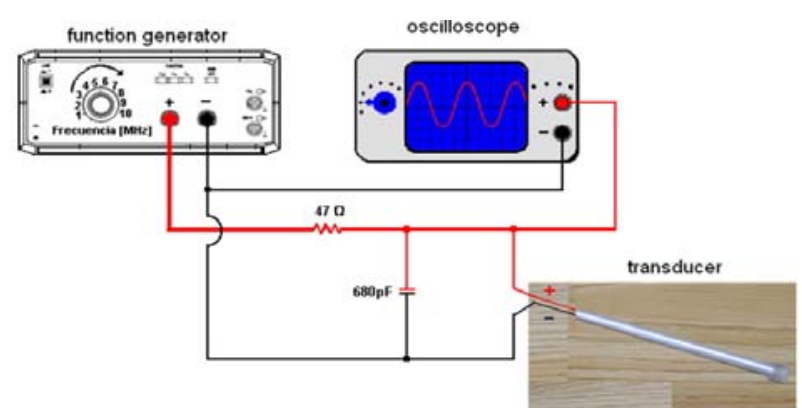

Fig. 4 Experimental set-up to find the resonance frequency of the transducer.

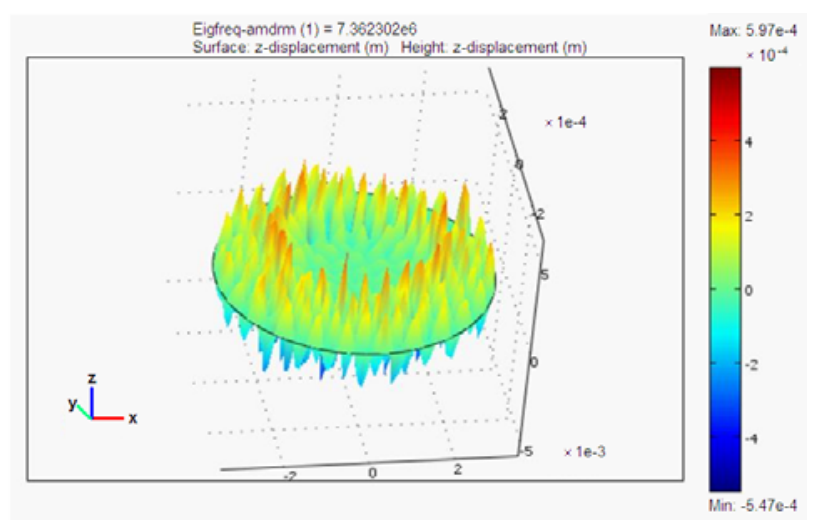

Fig. 5 Simulation of the piezoelectric ceramic using the finite elements method.

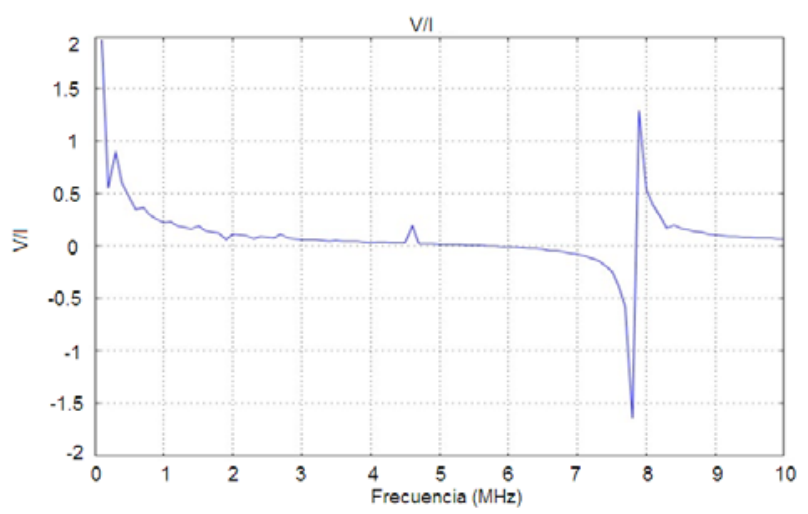

Fig. 6 Simulated impedance of the piezoelectric ceramic. 


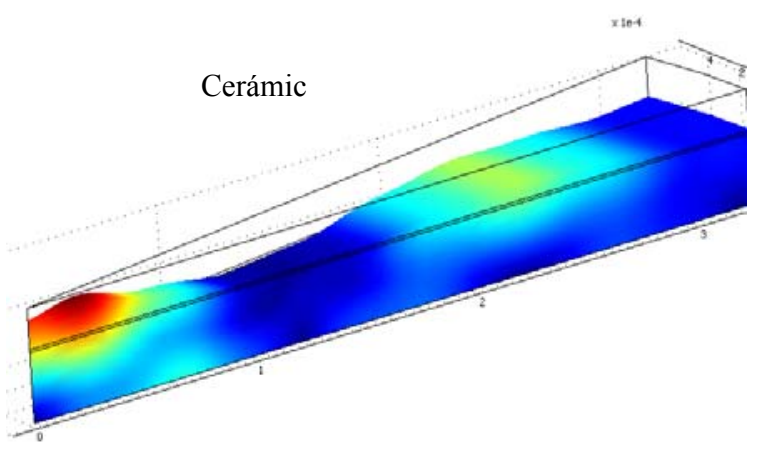

(a)

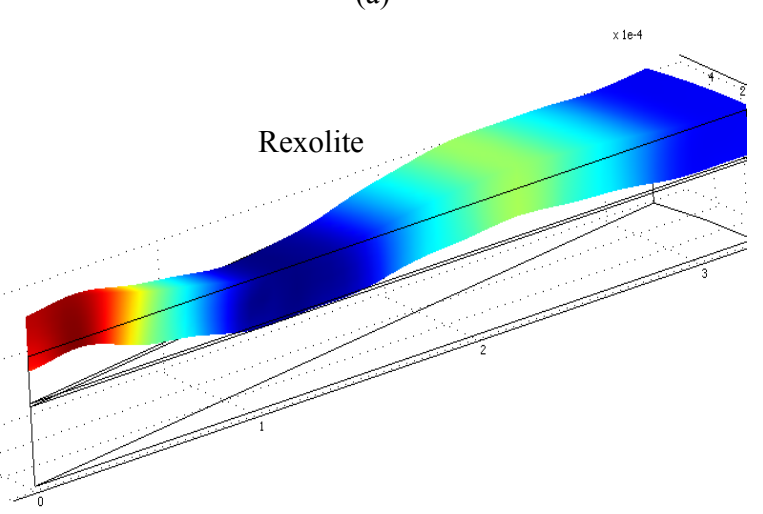

(b)

Fig. 7 Simulation of piezoelectric ceramic with matching of Rexolite. We present only a portion of the simulation. (a) the response of ceramics and (b) layer Rexoite response.

process is simpler than when the whole geometry is considered.

Fig. 8 shows the plot of the impedance, where it is possible to see that there is a frequency shift and this shift may be due to the thickness of the Rexolite layer, however, previous work $[3,11]$ shows that the thickness of the layer should not influence the acoustic matching.

Fig. 9 shows the simulation of ceramic and the Rexolite together oscillating, here, it is posible to see form of oscillation of both materials, as well as the components of mechanical stress that are formed in the Rexolite when the ceramic vibrates, it is important to mention that energy transfer is from the ceramic towards the Rexolite, although there is a good matching, the mechanical resistance of the Rexolite causes a mechanical tension, since the natural frequency of the ceramic does not correspond to that of the Rexolite.

The way that responds the ceramic with a Rexolite layer placed on it, produces a frequency shift as shown in Fig. 10. Fig. 11 shows the maximum displacement when there is a Rexolite layer, the resulting oscilation is according to the thickness of the layer and it is very clear to see the difference of oscillations when there is and where there is not a layer. These results indicate that it is necessary to correctly calculate the thickness

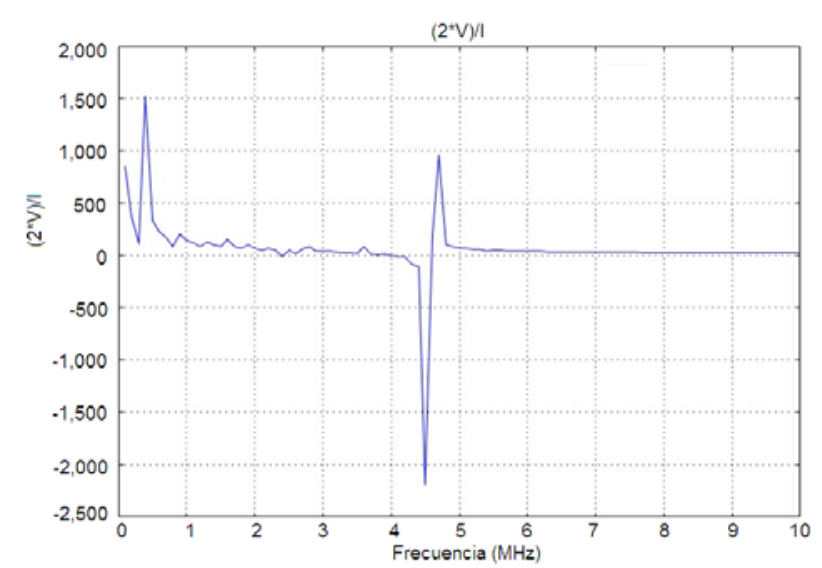

Fig. 8 simulation plot of the resonance frequency of the ceramic and the Rexolite Layer.

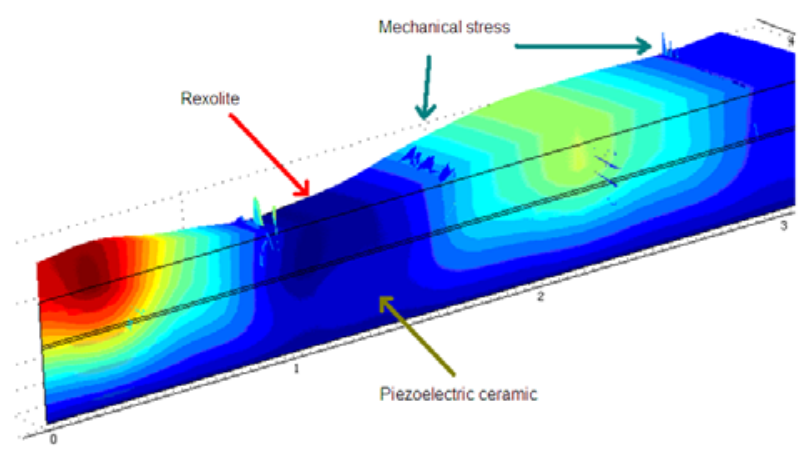

Fig. 9 Simulation of the ceramic and the Rexolite layer (mechanicalstress).

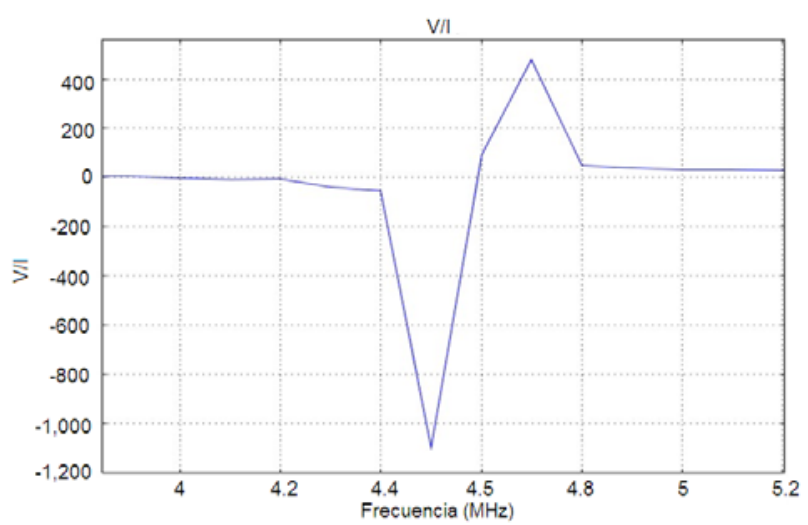

Fig. 10 Frequency response of the simulation of the ceramic and the Rexolite layer. 


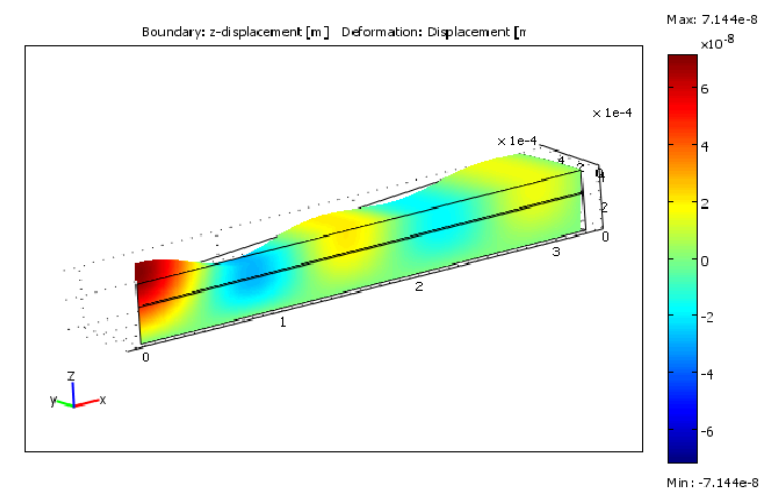

Fig. 11 Simulation of the maximum displacement of the ceramic and Rexolite layer.

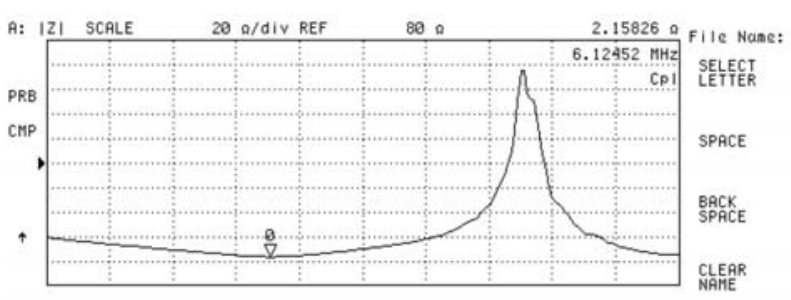

(a)

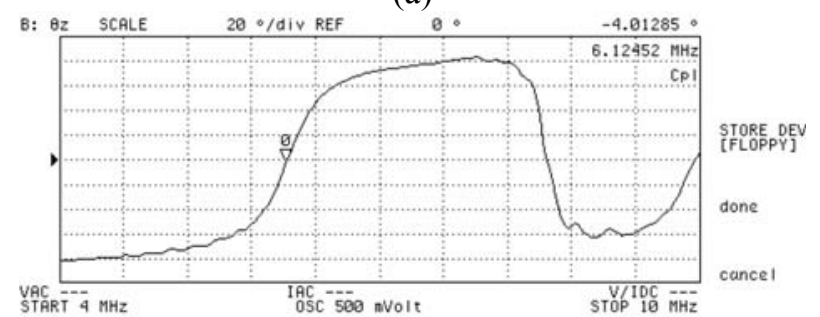

(b)

Fig. 12 Graphs represent the response curves for the prototype of the transducer, which resembled the oscillation frequency thereof. (a) the impedance curve corresponding to the (b) the phase response of the transducer.

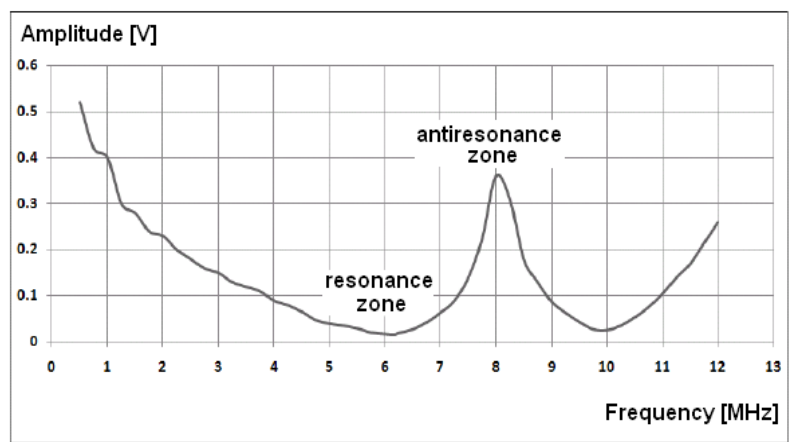

Fig. 13 Plot of the resonance frequency of the prototype transducer.

of the Rexolite layer to ensure a correct vibration frequency that serves for medical applications. To ensure that the phenomenon of acoustic matching is guaranteed in the simulation, we need that the common borders are united and have the same properties.

The experimental results for the prototype transducer are presented below.

Fig. 12 shows the response of the prototype transducer when was analyzed using the impedance meter. The value of the resonance frequency is $6.12452 \mathrm{MHz}$, this value is consistent with what we expected since the resonance frequency of the transducer must decrease due to the added Rexolite layer, as well as to the welded electrodes which produces a frequency shift. Despite this frequency shift, the operation range of the transducer is more than acceptable for our applications.

As already mentioned to obtain the value of the resonance frequency on an experimental basis, the equivalent circuit (shown in the Fig. 3) was replaced by the prototype transducer, the result is shown in Fig. 13. In this case the measured frequency was $6 \mathrm{MHz}$, this value is not so accurate due to the resolution of the oscilloscope, however, the measured value is quite close to that measured using the impedance analyzer.

The simulation and experimental results are very similar, this confirms that the transducer constructed works properly and may be useful for our applications, and also based on these results we can assure that the performance of the prototype transducer is very close to the simulated.

At last, it has been shown that the design and construction of ultrasonic transducers using the method described in this paper are a fact, providing a reliable tool for future applications.

\section{Conclusions}

The simulations presented in this work allowed us to determine the performance of the ceramic by itself and that of the ceramic with the Rexolite layer. It has been demonstrated that there is a shift in the resonance frequency when the Rexolite layer is taken into account; this is basically due to the thickness of the Rexolite layer used in the simulation. Eventhough there are results in previous works that show that Rexolite offers 
a good acoustic matching regardless of the thickness; it is recommended in the process of construction of a transducer that the layer is as thin as possible to ensure that there is no attenuation. Based on the results it is possible to ensure that the performance of a prototype transducer may be well predicted using the finite element method.

Although the shift in the resonance frequency indicates that it must be necessary to use ceramics at a higher frequency, so that the prototype transducer has a closer resonance frequency to that of the simulation $(8$ $\mathrm{MHz}$ ), overall results showed that the experimental resonce frequency is good enough for our applications at both the respective acoustic laboratories in Polytechnic University of Madrid and the National Autonomous University of Mexico

\section{Acknowledgments}

The authors thank the support of the DGAPA-UNAM (PAPIIT IN-113610). Also to AlßAN Office grant number IO4D00029MX

\section{References}

[1] I. Sánchez, J. Contreras, P. Acevedo, M. Fuentes, Finite Element Simulation Using an Ultrasonic Transducer Array type (Array) for Medical Applications, SOMI XXIII, Instrumentation Congress, Xalapa, Veracruz, México, octubre de 2008, memories CD.

[2] I. Sánchez, P. Acevedo, J. Contreras, Simulation and characterization of an ultrasonic transducer as the acoustic coupling using Rexolite, in: 29th Annual Meeting International Conference on Materials, Surface and Vacum, San Luis Potosi, México, 2009.

[3] I. Sánchez, P.Acevedo, M. Fuentes, The use of a novel acoustic matching material in the construction of an ultrasonic Doppler transducer for medical application, Pan American Health Care Exchanges, PAHCE, IEEE (2010) 3-7.

[4] Characteristics of rexolite, http://www.rexolite.com/.

[5] S. Domínguez, P.A. Contla, J.A. Contreras, M.F. Cruz, Simulation and construction of an ultrasonic transducer applied to blood flow detection, in: Pan American Health Care Exchanges, PAHCE, IEEE (2011) 144-147.

[6] Piezoelectric Materials: Typical Parameters of Piezoelectric Ceramics, http://www.physikinstrumente.com/.

[7] C.M. Rumack, Diagnostic Ultrasound, Editorial Grafos, 3th ed., Spain, 2006, pp. 21-26.

[8] J. Salazar, Contribution to improved resolution of imaging systems by ultrasound, Polytechnic University of Catalunya (1997) 6-7.

[9] P.A. Contla, I.S. Domínguez, A.Z. Vázquez, Improvement design and construction of an ultrasonic transducer applied to blood flow measurement, in: 1st International Congress on Instrumentation and Applied Sciences ICIAS (Incorporating the 25th National Congress on Instrumentation) Cancún, Quintana Roo, México, 2010, pp. 1-7.

[10] Manual COMSOL 3.3, Manual Structural Mechanics Module Version 3.3, Agosto, 2006, pp. 323-346.

[11] I.S. Domínguez, P.A. Contla, New test box for selecting materials based on their acoustic properties for constructing ultrasonic piezoelectric transducers, SOMI XXIV, Instrumentation Congress, Mérida, Yucatán, México, 2009. 